\title{
Cerebrovascular Physiology
}

National Cancer Institute

\section{Source}

National Cancer Institute. Cerebrovascular Physiology. NCI Thesaurus. Code C18691.

A medical specialty focusing on the normal blood circulation of the brain and on changes due to pathology. 